\title{
Embolic Brain Abscess in a Patient With Tricuspid Valve Endocarditis and a Patent Foramen Ovale
}

\author{
Gregory Suero ${ }^{\mathrm{a}, \mathrm{b}}$, Rekhi Varghese ${ }^{\mathrm{a}}$, Moneal Shah ${ }^{\mathrm{a}}$, David Lasorda ${ }^{\mathrm{a}}$
}

\begin{abstract}
Brain abscess results from local or metastatic septic spread to the brain. Approximately, $15-60 \%$ of brain abscesses are considered to be cryptogenetic, being the primary infectious site often undetected. Patent foramen ovale (PFO) has been suggested as a potential source of paradoxical embolism; this was suggested after detecting a higher prevalence of PFO in ischemic stroke of unexplained cause. Brain abscess may develop as a consequence of paradoxical infectious emboli from an extracranial infective source. We present the case of a patient with no history of intravenous drug use and no periodontal disease, which was found to have a brain abscess, tricuspid valve endocarditis and the presence of a PFO with right to left shunting with no other source of infection.
\end{abstract}

Keywords: Brain abscess; Infective endocarditis; Paradoxical emboli; Patent foramen ovale; Tricuspid valve; Percutaneous closure

\section{Introduction}

Neurological complications occur in 20 to $40 \%$ of patients with infective endocarditis and represent a major factor associated with increased mortality [1]. Dissemination of emboli into cerebral or meningeal vessels can lead to brain abscess or meningitis [2]. Brain abscesses are commonly associated with contiguous infective focus such as otitis media or sinusitis, hematogenous spread from a distant focus or cranial

Manuscript accepted for publication June 18, 2012

${ }^{a}$ Department of Cardiology, Allegheny General Hospital; Pittsburgh, PA. 15224, Dr. Varghese is now part of the Department of Cardiology, St. Elizabeth Health Center; Youngstown, OH.44504, USA

${ }^{\mathrm{b}}$ Corresponding author: Gregory Suero, Department of Interventional Cardiology, Allegheny General Hospital, 320 East North Av, 4th Floor Snyder Pavilion. Pittsburgh, PA. 15212, USA.

Email: gsuero@wpahs.org

doi:10.4021/jnr116w trauma [3, 4]. Patent foramen ovale (PFO) can act as an avenue for right-to-left shunt bypassing the pulmonary circulation, and it has been associated with cryptogenic cerebrovascular events [5]. There have been cases reports of patients with PFO who presented with brain abscess that was thought to arise from periodontal disease [6]. In other case reports, no source of infection was identified. Percutaneous closure of the PFO has been suggested to be effective in the prevention of recurrent cerebrovascular events $[7,8]$ and brain abscesses [9].

\section{Case Report}

A 43 years old female with no significant past medical history presented to be the emergency department with acute onset of numbness and weakness involving her right hemi body. She was in her usual state of health and denied any recent fevers, chills, headaches, nausea, vomiting, visual changes, diarrhea, abdominal pain, cough, syncope or seizure like activity. She denied any recent hospitalizations and was not on any outpatient medications. Her family and social history was unremarkable, including no history of recreational drug use, no recent travel, no sick contacts, no recent dental procedures and no pets. Her vital signs on admission and cardiac examination were within normal limits. Neurological examination revealed decreased sensation in the right side of her face, right upper and lower extremities; otherwise, she had intact cranial nerves and higher motor function, pupils that were equal and reactive bilaterally, symmetrical reflexes, normal down going plantars and no visual fields deficits. Initial CT scan of the head revealed a $5 \mathrm{~mm} \times 1 \mathrm{~cm}$ lesion in the left frontoparietal area with surrounding edema. MRI of the brain with and without contrast revealed a $1.1 \times 1.1 \times 1.0$ $\mathrm{cm}$ peripherally enhancing lesion in the superior aspect of the left precentral gyrus with surrounding edema with mild enhancement of the surrounding dura suspicious for malignancy (Fig. 1a). There was no radiologic evidence of otitis, mastoiditis or sinusitis. Since most of her symptoms had resolved, she was discharged home with plans to proceed with an outpatient surgical evaluation and elective surgical removal of the presumed neoplasm. 

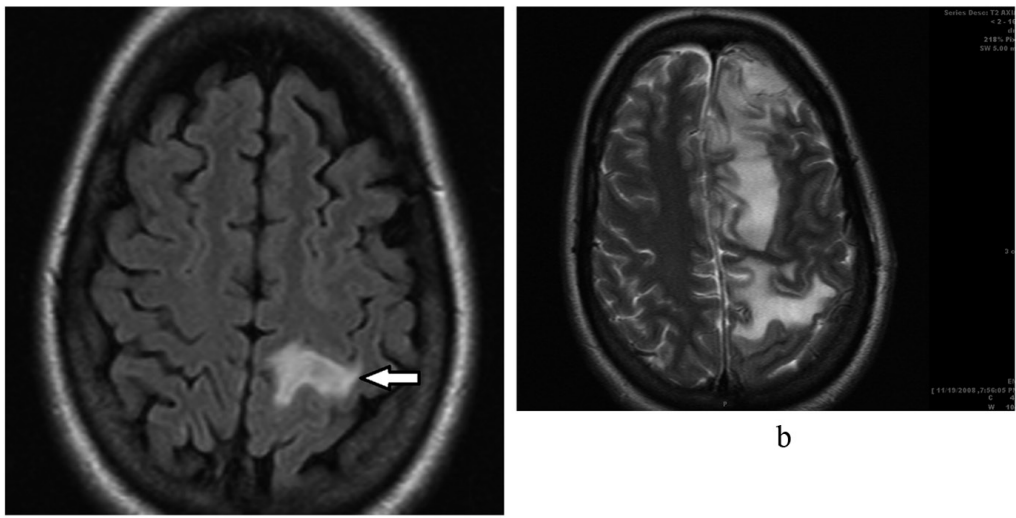

$\mathrm{b}$

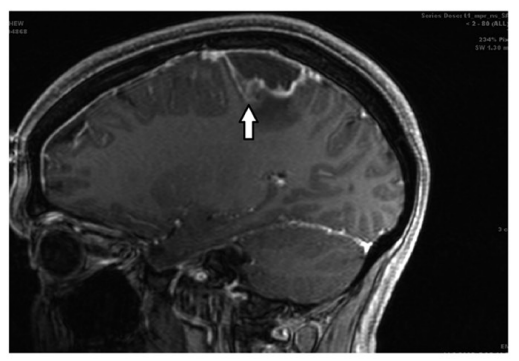

Figure 1. a. Brain MRI shows $1.1 \times 1.1 \times 1.0 \mathrm{~cm}$ peripherally enhancing lesion (arrow) in the superior aspect of the left precentral gyrus with surrounding edema and mild enhancement of the surrounding dura. b. Repeat brain MRI shows a worsening appearance with thickened enhancing pachymeninges of the superior frontal and parietal region as well as diffuses leptomeningeal enhancement and increasing mass effect. There was significant increased abnormal T2 signal throughout the superior left cerebral hemisphere consistent with vasogenic edema. c. Midline sagittal cut showing brain abscess (arrow) with significant surrounding edema.

The patient returned to the emergency room ten days later with fevers, right sided weakness, headaches, nausea, vomiting and leukocytosis. Repeat MRI of the head revealed diffuse superior left sided subdural empyema and meningitis with associated vasogenic edema and increased mass effect (Fig. 1b, 1c); with clear sinuses and no mandibular abscess. Due to the high suspicion for a brain abscess, the patient underwent emergent craniotomy. During surgery, when the meninges were retracted, frank pus was seen draining from the operative wound. After extensive saline irrigation, the wound was closed and the patient transferred to the neurosurgical intensive care unit. The patient was treated with broad spectrum antibiotics and antiepileptics. Gram stain of the specimen revealed gram positive cocci and the culture grew streptococcus viridans.

A search for the primary source of infection led to an extensive workup that included blood and urine cultures, human immunodeficiency virus, tuberculosis, rheumatology panel, and computed tomography of the chest, abdomen and pelvis, all of which returned normal results. The patient had a transesophageal echocardiogram, which showed a large amorphous mobile echodensity arising from the anterior leaflet of the tricuspid valve suggestive of endocarditis (Fig.
2).

A PFO with right to left shunt demonstrated by color Doppler (Fig. 3) and agitated saline contrast injection was also noted. The patient had a satisfactory recovery and was sent to a rehabilitation facility on intravenous antibiotic therapy. The follow-up MRI scan 2 months later showed nearcomplete resolution of the brain abscess and she experienced a complete clinical recovery.

\section{Discussion}

Brain abscesses are rarely encountered in clinical practice and the incidence has been noted to be about 1 case per 100,000 in the general population $[10,11]$. Several potential mechanisms have been described including expansion from a contiguous focus (e.g., sinusitis or otitis media), hematogenous spread from arterial circulation (e.g., endocarditis or lung abscess) and direct infection from cerebral trauma [12]. However 15 to $30 \%$ of the reported cases had no primary infectious source identified and are thought to be cryptogenic $[3,13]$. Many of these cryptogenic abscesses are thought to be secondary to asymptomatic dental infections $[12,14,15]$. 


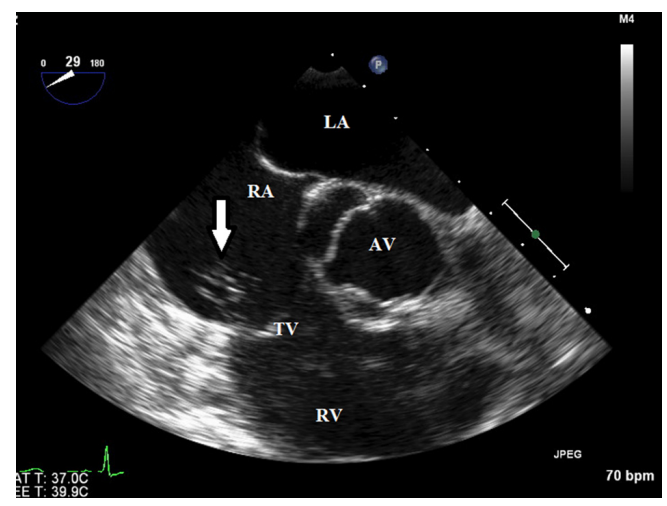

Figure 2. Transesopagheal echocardiogram showed a large amorphous mobile echodensity arising from the anterior tricuspid valve leaflet (arrow) highly suspicious for a vegetation.

Kawamata et al proposed an alternate mechanism by which flora commonly found in the oropharynx could cause a brain abscess by paradoxical embolism through a PFO secondary to a right to left shunt [4].

The prevalence of PFO in the general population is relatively high. In autopsy series, the prevalence ranged from 20 to $35 \%$, with a decrease in prevalence and an increase in size with increasing age [16]. With increasing use of TEE, a prevalence of $26 \%$ has been reported, which is almost the same as the frequency found by autopsy in the general population [17]. Frequent forms of presentation of paradoxical embolism include cryptogenic stroke, peripheral embolism and decompression sickness in divers. Similarly, paradoxical embolism through a PFO represents a potential mechanism of brain abscess development via hematogenous spread. In fact, PFO has been implicated in brain abscesses anywhere from 4 to $26 \%$ of cases attributed to congenital heart disease [18]. Furthermore, on the basis of a reported series in a patient group with no significant predisposing risk factors, cerebral abscess may in itself be a risk factor for recurrent abscess [19]. Percutaneous closure of the PFO has been demonstrated to be effective in a number of conditions thought to be related to right to left shunting including cryptogenic strokes, decompression sickness and platypnea-orthodexia syndrome [20].

Tricuspid valve infective endocarditis (TVIE) is rare and accounts for $5 \%$ to $10 \%$ of infective endocarditis. Isolated native non-rheumatic TVE is very rarely diagnosed in the absence of intravenous drug use, congenital heart disease, insertion of a central venous catheter, placement of a pacemaker or implantable defibrillator or hemodialysis. The reasons why TVIE is rare are thought to be because in the right-sided system, congenital heart disease is infrequent; the tricuspid and pulmonary valves are not strained because of low pressure; and oxygen saturation is low. The clinical presentation of patients with TVIE differs from those with left-sided endocarditis, as these patients often do not have a

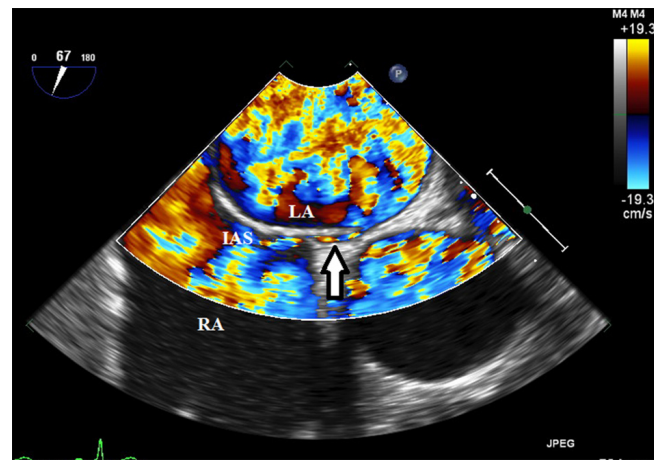

Figure 3. A small Patent foramen ovale (arrow) was demonstrated by color Doppler and agitated saline contrast injection.LA: left atrium; RA: right atrium; IAS: interatrial septum; AV: aortic valve; TV: tricuspid valve.

detectable heart murmur and the peripheral manifestations, such as splinter or conjunctival hemorrhages, are virtually absent. Heart failure is unusual in these patients as well.

\section{Conclusions}

Our case describes a rare case of a patient with no predisposing factors diagnosed with a solitary brain abscess secondary to isolated tricuspid valve endocarditis and a PFO. We believe that patients with a brain abscess from an unknown source should be screened with a transesophageal echocardiogram. These patients should then undergo surgical evacuation of the abscess in association with prolonged antibiotic therapy. It is uncertain if patients with a PFO, TVIE and brain abscess of unknown or undetermined source of infection would benefit from PFO closure. In our case, the etiology was felt to be TVE and treatment would prevent further embolic events or seeding. Only a randomized trial of PFO closure may answer this question, but it would be difficult due to the rare nature of this clinical scenario.

\section{Conflict of Interest}

The authors declare no conflict of interest.

\section{References}

1. Mylonakis E, Calderwood SB. Infective endocarditis in adults. N Engl J Med. 2001;345(18):1318-1330.

2. Hoen B, Alla F, Selton-Suty C, Beguinot I, Bouvet A, Briancon S, Casalta JP, et al. Changing profile of infective endocarditis: results of a 1-year survey in France. JAMA. 2002;288(1):75-81.

3. Wispelwey B, Dacey RG Jr, Scheld WM. Infections of the Central nervous System. Philadelphia, Lippincott- 
Raven publishers, 1997, Ed 2, pp 463-493.

4. Kawamata T, Takeshita M, Ishizuka N, Hori T. Patent foramen ovale as a possible risk factor for cryptogenic brain abscess: report of two cases. Neurosurgery. 2001;49(1):204-206; discussion 206-207.

5. Lechat P, Mas JL, Lascault G, Loron P, Theard M, Klimczac M, Drobinski G, et al. Prevalence of patent foramen ovale in patients with stroke. $\mathrm{N}$ Engl J Med. 1988;318(18):1148-1152.

6. Khouzam RN, El-Dokla AM, Menkes DL. Undiagnosed patent foramen ovale presenting as a cryptogenic brain abscess: case report and review of the literature. Heart Lung. 2006;35(2):108-111.

7. Martin F, Sanchez PL, Doherty E, Colon-Hernandez PJ, Delgado G, Inglessis I, Scott N, et al. Percutaneous transcatheter closure of patent foramen ovale in patients with paradoxical embolism. Circulation. 2002;106(9):11211126.

8. Braun M, Gliech V, Boscheri A, Schoen S, Gahn G, Reichmann H, Haass M, et al. Transcatheter closure of patent foramen ovale (PFO) in patients with paradoxical embolism. Periprocedural safety and mid-term followup results of three different device occluder systems. Eur Heart J. 2004;25(5):424-430.

9. LaBarbera M, Berkowitz MJ, Shah A, Slater J. Percutaneous PFO closure for the prevention of recurrent brain abscess. Catheter Cardiovasc Interv. 2006;68(6):957-960.

10. Nicolosi A, Hauser WA, Musicco M, Kurland LT. Incidence and prognosis of brain abscess in a defined population: Olmsted County, Minnesota, 1935-1981. Neuroepidemiology. 1991;10(3):122-131.

11. Nunez DA, Browning GG. Risks of developing an otogenic intracranial abscess. J Laryngol Otol. 1990;104(6):468-472.

12. Kastenbauer S, Pfister HW, Wispelwey B, Scheld WM. Brain abscess. Infections on the central nervous system. 3rd ed. Philadelphia: Lipincott Williams \& Williams; 2004. 479-507.

13. Mathisen GE, Johnson JP. Brain abscess. Clin Infect Dis. 1997;25(4):763-779; quiz 780-761.

14. Schuman NJ, Turner JE. Brain abscess and dentistry: a review of the literature. Quintessence Int. 1994;25(6):411413.

15. Li X, Tronstad L, Olsen I. Brain abscesses caused by oral infection. Endod Dent Traumatol. 1999;15(3):95101.

16. Hagen PT, Scholz DG, Edwards WD. Incidence and size of patent foramen ovale during the first 10 decades of life: an autopsy study of 965 normal hearts. Mayo Clin Proc. 1984;59(1):17-20.

17. Konstadt SN, Louie EK, Black S, Rao TL, Scanlon P. Intraoperative detection of patent foramen ovale by transesophageal echocardiography. Anesthesiology. 1991;74(2):212-216.

18. Yang SY. Brain abscess associated with congenital heart disease. Surg Neurol. 1989;31(2):129-132.

19. Yousaf I, McInstry CS, Choudhari KA. Recurrent intracranial abscess after a long latency. Br J Neurosurg. 2004;18(4):396-397.

20. Wilmshurst PT, Nightingale S, Walsh KP, Morrison WL. Effect on migraine of closure of cardiac right-toleft shunts to prevent recurrence of decompression illness or stroke or for haemodynamic reasons. Lancet. 2000;356(9242):1648-1651. 